\title{
MicroRNA-127-3p promotes glioblastoma cell migration and invasion by targeting the tumor-suppressor gene SEPT7
}

\author{
HUAWEI JIANG ${ }^{1,2}$, DASONG HUA ${ }^{2}$, JING ZHANG $^{2}$, QING LAN $^{3}$, QIANG HUANG ${ }^{3}$, JAE-GEUN YOON ${ }^{4}$, \\ XU HAN ${ }^{2}$, LISHA LI ${ }^{2}$, GREGORY FOLTZ ${ }^{4 *}$, SHU ZHENG ${ }^{1}$ and BIAOYANG LIN ${ }^{1,2,4,5}$ \\ ${ }^{1}$ Cancer Institute (Key Laboratory of Cancer Prevention and Intervention, China National Ministry of Education), \\ The Second Affiliated Hospital, Zhejiang University School of Medicine, Hangzhou, Zhejiang 310009; \\ ${ }^{2}$ Systems Biology Division, Zhejiang-California International Nanosystems Institute, Zhejiang University, \\ Hangzhou, Zhejiang 310029; ${ }^{3}$ Department of Neurosurgery and Brain Tumor Research Laboratory, \\ Second Affiliated Hospital of Soochow University, Suzhou, Jiangsu 215004, P.R. China; \\ ${ }^{4}$ Swedish Neuroscience Institute, Swedish Medical Center, Seattle, WA 98122; \\ ${ }^{5}$ Department of Urology, University of Washington, Seattle, WA 98195, USA
}

Received December 15, 2013; Accepted February 6, 2014

DOI: $10.3892 /$ or.2014.3055

\begin{abstract}
MicroRNAs (miRNAs) are small non-coding RNAs of 20-25 nucleotides in length that are capable of modulating gene expression post-transcriptionally. The potential roles of miRNAs in the tumorigenesis of glioblastoma (GBM) have been under intensive studies in the past few years. In the present study, we found a positive correlation between the levels of miR-127-3p and the cell migration and invasion abilities in several human GBM cell lines. We showed that miR-127-3p promoted cell migration and invasion of GBM cells using in vitro cell lines and in vivo mouse models. We identified SEPT7, a known tumor-suppressor gene that has been reported to suppress GBM cell migration and invasion, as a direct target of miR-127-3p. SEPT7 was able to partially abrogate the effect of miR-127-3p on cell migration and invasion. In addition, microarray analysis revealed that miR-127-3p regulated a number of migration and invasionrelated genes. Finally, we verified that miR-127-3p affected
\end{abstract}

Correspondence to: Professor Shu Zheng, Cancer Institute (Key Laboratory of Cancer Prevention and Intervention, China National Ministry of Education), The Second Affiliated Hospital, Zhejiang University School of Medicine, Hangzhou, Zhejiang 310009, P.R. China

E-mail: zhengshu@zju.edu.cn

Dr Biaoyang Lin, Division of Systems Biology, Zhejiang-California International Nanosystems Institute (ZCNI), Zhejiang University, 268 Kaixuan Road, Hangzhou, Zhejiang 310029, P.R. China

E-mail: biaoylin@gmail.com

\section{"Deceased}

Key words: miR-127-3p, SEPT7, glioblastoma, migration the remodeling of the actin cytoskeleton mediated by SEPT7 in GBM cells.

\section{Introduction}

Gliomas are the most malignant and prevalent brain tumors of glial origin and are divided into four clinical grades on the basis of their histology and prognosis. Grade IV GBM is the most malignant of all brain tumors. Despite recent advances in diagnostics and treatments, the prognosis of advanced patients suffering from GBM remains poor (1). One of the most important reasons for this dismal outcome is that the migration and invasion of GBM cells eventually lead to tumor recurrence and patient death. However, the factors that mediate GBM migration and invasion are still poorly understood.

MicroRNAs are a class of 20-25 nucleotide long non-coding RNAs that modulate gene expression post-transcriptionally by regulating mRNA translation or stability (2). The human miR-127 gene is located within a cluster on chromosome 14q32.31. The hsa-miR-127 precursor can express two mature miRNAs, miR-127-3p and miR-127-5p. Changes in the expression of miR-127 were previously observed in esophageal squamous cell carcinoma (3) and medullary thyroid carcinoma (4), and miR-127 was proposed to be a tumor suppressor in human bladder cancer cells (5). However, the role of miR-127 in GBM development has not been well documented and little is known in regards to its target genes.

We decided to investigate the roles of miR-127-3p in GBM. We found that miR-127-3p promoted cell migration and invasion using in vitro cell lines and in vivo mouse models. We further demonstrated that SEPT7 is a direct target of miR$127-3 p$ and partially mediated the process of cell migration and invasion initiated by miR-127-3p. In addition, we performed microarray analysis after miR-127-3p overexpression was induced in a GBM cell line and found that the expression of many migration and invasion-related genes was significantly altered. Finally, immunofluorescent cell staining showed that 
miR-127-3p affected the remodeling of the actin cytoskeleton by regulating SEPT7 in GBM cells.

\section{Materials and methods}

Cell culture and reagents. Human GBM cell lines, LN229, T98G, A172, U87 and U251, were obtained from ATCC (http://www.atcc.org/) and grown in Dulbecco's Modified Eagle's medium (DMEM) supplemented with $10 \%$ fetal bovine serum (FBS). Cells were incubated at $37^{\circ} \mathrm{C}$ and supplemented with $5 \% \mathrm{CO}_{2}$. Antibodies specific to SEPT7 and GAPDH were obtained from Santa Cruz Biotechnology (Santa Cruz, CA, USA). Horseradish peroxidase-coupled secondary antibodies were purchased from MultiSciences Biotech Co., Ltd. (Hangzhou, China). miR-127-3p inhibitors, which are antisense sequences of miR-127-3p with 2'-O-methyl modification for in vitro transfection, and their respective negative controls were obtained from Guangzhou RiboBio Co., Ltd. (Guangzhou, China).

Plasmid construction and establishment of stable miR-127-3p-overexpressing cells. The genomic sequence of the human miR-127-3p gene was PCR amplified using the primer5'-GGAAGATCTGTAGTCCTGTCTGTTGGTCAG-3' and 5'-CCC AAGCTTCCTGAAGAACTGCTTCCGCC-3' from LN229 cells and cloned into pSUPER.neo (Oligo Engine, Seattle, WA, USA). It was designated as pSUPER.neo-miR127-3p. Lipofectamine 2000 (Invitrogen, Carlsbad, CA, USA) was used for the transfection of the DNA plasmid according to the manufacturer's protocol. Positive cells selected with $1 \mathrm{mg} /$ ml G418 were confirmed by stem-loop RT-PCR. The fulllength 3'UTR of SEPT7 was subcloned into the pmirGLO vector (Promega, Madison, WI, USA) to generate pmirGLOSEPT7-3'UTR. Mutant construct of SEPT7-3'UTR, named pmirGLO-SEPT7-3'UTR-mut, which carried a substitution of 4 nucleotides within the core binding site of SEPT7-3'UTR, was carried out using Takara MutanBEST kit. The coding sequence of SEPT7 was synthesized and subcloned into the pUC57-simple vector (GenScript, Piscataway, NJ, USA), and then was amplified by PCR and subcloned into the pcDNA3.1 vector, named pcDNA3.1-SEPT7. The primers for SEPT73'UTR were 5'-CCGCTCGAGCTCTCTATTGACCACCAG TTAACG-3' and 5'-AGCTTTCCTGCAGGCAGTGCCAAT ATATGGAAAATATC-3'. The primers for mutation were 5'-TAGGGTTTGACCAATTTGCACCAGTTTTATCC-3' and 5'-CCAACAAACACTGATGTCCAAGCTGGC-3'. All PCR products were verified by DNA sequencing.

RNA quantification. Total RNA, containing miRNA, was extracted with TRIzol reagent (Invitrogen) following the manufacturer's instructions. For mRNA analysis, reverse transcription was performed by using M-MLV reverse transcriptase (Promega). Amplification reactions were performed using the SYBR ${ }^{\circledast}$ Premix Ex Taq ${ }^{\mathrm{TM}}$ (Takara, Dalian, China). Data were normalized to the level of glyceraldehyde-3-phosphate dehydrogenase expression in each sample. Expression of miR-127-3p was quantified using stem-loop RT-PCR analysis as reported (6). The primers and other reagents for stem-loop RT-PCR were obtained from Guangzhou RiboBio. U6 RNA was used as an internal control. The $2^{-\Delta \Delta C t}$ method for relative quantification of gene expression was used to determine the expression levels of the transcripts.

Migration and invasion assays. For the migration assay, cells were plated onto 24-well Transwell chambers (Corning, Corning, NY, USA) with an $8-\mu \mathrm{m}$ pore polycarbonate membrane. For the invasion assay, cells were plated on chambers precoated with ECM gel (Sigma, St. Louis, MO, USA). In both assays, the cells were plated in medium without serum, and medium containing 10\% FBS in the lower chamber served as a chemoattractant. After $24 \mathrm{~h}$, the cells on the upper surface were scraped and washed away, whereas the cells on the lower surface were fixed and stained with $0.05 \%$ crystal violet. Finally, six visual fields for each insert were randomly selected and counted under a microscope. The cell numbers were normalized to the control cell number, which was set to 100 . The results were averaged from three independent experiments.

Animal model. Five-week-old female nude mice were purchased from Zhejiang Chinese Medical University (Zhejiang, China). All experimental protocols were approved by the Ethics Committee for Animal Experimentation of Zhejiang University. Exponentially growing LN229 cells $\left(2 \times 10^{6}\right)$ with stable ectopic expression of miR-127-3p or control vector were injected into nude mice through the tail vein $(n=5)$. Eight weeks after injection, the animals were sacrificed. The liver and lung tissues were removed, sectioned, and stained with hematoxylin and eosin (H\&E) stain to count the tumor loci by a pathologist.

Luciferase assay. LN229 cells were transiently transfected with the luciferase reporter gene constructs and miR-127-3p-expressing plasmid, and U251 cells were transiently transfected with the luciferase reporter gene constructs and miR-127-3p inhibitors. After $48 \mathrm{~h}$, luciferase activities were measured using a dual luciferase reporter assay system according to the manufacturer's protocol (Promega). For each plasmid construct, three independent transfection experiments were performed in triplicate.

Western blot analysis. The cells were lysed by a standard procedure in radioimmunoprecipitation assay buffer containing protease inhibitor cocktail (Calbiochem, Darmstadt, Germany). Protein concentrations of total cell lysates were measured using a BCA protein assay kit (Pierce Biotechnology, Rockford, IL, USA). Protein was then separated on polyacrylamide gels, transferred to a nitrocellulose membrane, incubated with the relevant antibodies, and detected with HRP-conjugated antibodies. Bands were visualized using ECL Western blotting detection reagents (Thermo Fisher Scientific, Rockford, IL, USA).

Microarray analysis. Two separate total RNA samples were extracted from LN229 cells with miR-127-3p overexpression and corresponding control cells. Genes regulated by miR-127-3p were identified by Affymetrix U133 Plus 2 GeneChip (Affymetrix, Santa Clara, CA, USA). The CEL files were imported into the Affymetrix's Expression Console (V1.1) program, and the data were normalized using 
RMA normalization. Then the data were exported to the GeneSpring Program (Agilent, Inc., Palo Alto, CA, USA) for further analysis. In order to remove the low-expressed genes in the chips, probes with intensities $<100$ in both chips were excluded. Genes with absolute change of $\geq 2$-fold and $\mathrm{P}<0.05$ were considered differentially regulated by miR-127-3p. The online High-Throughput GoMiner program was used to analyze the Gene Ontology (GO) using biological processes terms at level 4. U251 GBM cells naturally present higher expression of miR-127-3p (as shown in Fig. 1), its array data (GSM803632) was downloaded from the GEO database on NCI 60 cell lines (http://www.ncbi.nlm.nih.gov/geo/query/acc. cgi?acc=GSE32474). The same analysis pipeline was used to identify differentially expressed genes between LN229 with miR-127-3p and U251.

Immunofluorescence cell staining. Cells were seeded on coverslips and incubated for $24 \mathrm{~h}$ and then fixed with formalin for $15 \mathrm{~min}$, washed with PBS, permeabilized for $3 \mathrm{~min}$ at room temperature with PBS containing $0.25 \%$ Triton X-100 and blocked for 30 min with PBS containing $1 \%$ of BSA and $0.5 \%$ Tween-20. Slides were incubated overnight at $4^{\circ} \mathrm{C}$ in a blocking solution containing anti-SEPT7 (Santa Cruz Biotechnology), washed with PBS, and then incubated in a blocking solution containing the secondary antibody conjugated with $\mathrm{Cy} 3$ (red) (Millipore, Darmstadt, Germany) and phalloidin-FITC (Beyotime Institute of Biotechnology, Haimen, China) for $1 \mathrm{~h}$. After washing, coverslips were attached to glass slides. Cells were imaged using a fluorescence microscope.

Statistical analysis. Data are presented as means \pm SD. Statistical comparisons of the studies were analyzed by Student's t test or Chi-square test as appropriate. Correlations between groups were calculated with Pearson's correlation analysis. A P value $<0.05$ was defined as indicative of a statistically significant result.

\section{Results}

miR-127-3p promotes human GBM cell migration and invasion. We initially analyzed the expression levels of miR-127-3p in five human GBM cell lines and found that there was great heterogeneity in miR-127-3p expression in the different GBM cell lines. Furthermore, we observed a statistically significant positive correlation between miR-127-3p levels and the cell migration or the cell invasion potential of the five GBM cell lines ( $\mathrm{r}=0.89$ and 0.93 , respectively; $\mathrm{P}<0.05$ ) (Fig. 1A). We observed a significant difference between the miR-127-3p high-expressing cell lines (U251 and U87) and low-expressing cell lines (LN229, A172 and T98 cells) in regards to migration and invasion potential $(\mathrm{P}=0.017$ and 0.011 , respectively; Student's t-test).

To determine whether miR-127-3p is merely correlated or directly regulates human GBM cell migration and invasion, we selected LN229 and T98G cells, which are two cell lines with low endogenous miR-127-3p expression, and U251 cells, which have a high endogenous miR-127-3p expression, for further analyses. Fig. $1 \mathrm{~B}$ and $1 \mathrm{E}$ show that we were able to induce miR-127-3p overexpression in the LN229 and T98G cells. We observed a $>2$-fold increase in cell migration and invasion potential in the LN229 and T98G cells with stable overexpression of miR-127-3p compared to the control cells (Fig. 1C, D, F and G).

In addition, we performed a complementary analysis by silencing miR-127-3p with an miR-127-3p inhibitor in U251 cells with high endogenous miR-127-3p expression (Fig. 1H). Inhibition of miR-127-3p in U251 cells led to $\sim 40-50 \%$ reduction in migration and invasion compared with the control cells (Fig. 1I and J).

miR-127-3p increases tumor formation in a mouse model. To determine the effects of miR-127-3p on tumor invasive potential in vivo, LN229 cells either overexpressing miR-127-3p or transfected with a control vector were injected via the tail vein into nude mice. Eight weeks after injection, the animals were sacrificed. The liver and lung tissues were removed, sectioned, and stained with H\&E to count the tumor loci by a pathologist.

In the mice inoculated with miR-127-3p-overexpressing cells, 5 of 5 cases had tumor formation in the liver and 2 of 5 cases had tumor formation in the lung. In contrast, 4 of 5 cases had tumor formation in the liver and none had tumor formation in the lung in the control group, both confirmed by pathohistological examinations (Fig. 2A and B). Although the number of tumors formed was higher in the miR-127-3p-overexpressing tumor group compared to the control cell group, the number was not statistically significant $(\mathrm{P}=0.29$ and 0.11 , respectively). However, as shown in Fig. $2 \mathrm{C}$, the average number of tumors formed in the liver in the miR-127-3p-overexpressing group was 42 , while it was 5 for the vector control group $(\mathrm{P}=0.02)$.

miR-127-3p targets the SEPT7 gene and its effects on cell migration and invasion are partially mediated through SEPT7. To explore the molecular mechanism of miR-127-3p function, we used the EIMMo program (http://www.mirz. unibas.ch/ElMMo2), which combined mRNA expression and targeted prediction algorithm, to predict targets of miR-127-3p in the human. We used the whole brain mRNA expression data set on the server to help predict miR-127-3p. One hundred and fifty-eight predicted targets were found. We found that SEPT7 had two entries (variant 1 and variant 2) that were among the top 10 predicted targets. Fig. 3A shows a miR-127-3p core binding site at nucleotides 67-74 of the SEPT7 3'UTR. In addition, the miR-127-3p target sequence at nucleotides $67-74$ of the SEPT7 3'UTR is highly conserved among different species (Fig. 3A). We then decided to conduct additional analysis to determine whether SEPT7 is a target of miR-127-3p.

We constructed pmirGLO-SEPT7-3'UTR and pmirGLOSEPT7-3'UTR-mut the latter contains mutated targeted sequences. Cotransfection of LN229 cells with pmirGLOSEPT7-3'UTR and pSUPER.neo-miR-127-3p caused a $22 \%$ decrease in the luciferase activity compared with the negative control. Four-nucleotide substitution in the core binding site (pmirGLO-SEPT7-3'UTR-mut) abolished the suppressive effects (Fig. 3B). In addition, pmirGLO-SEPT7-3'UTR and pmirGLO-SEPT7-3'UTR-mut were cotransfected with either miR-127-3p inhibitor or the control oligonucleotide into U251 cells, expressing high levels of endogenous miR127-3p. The inhibition of miR-127-3p by the inhibitors resulted in a significant increase in luciferase activity of 


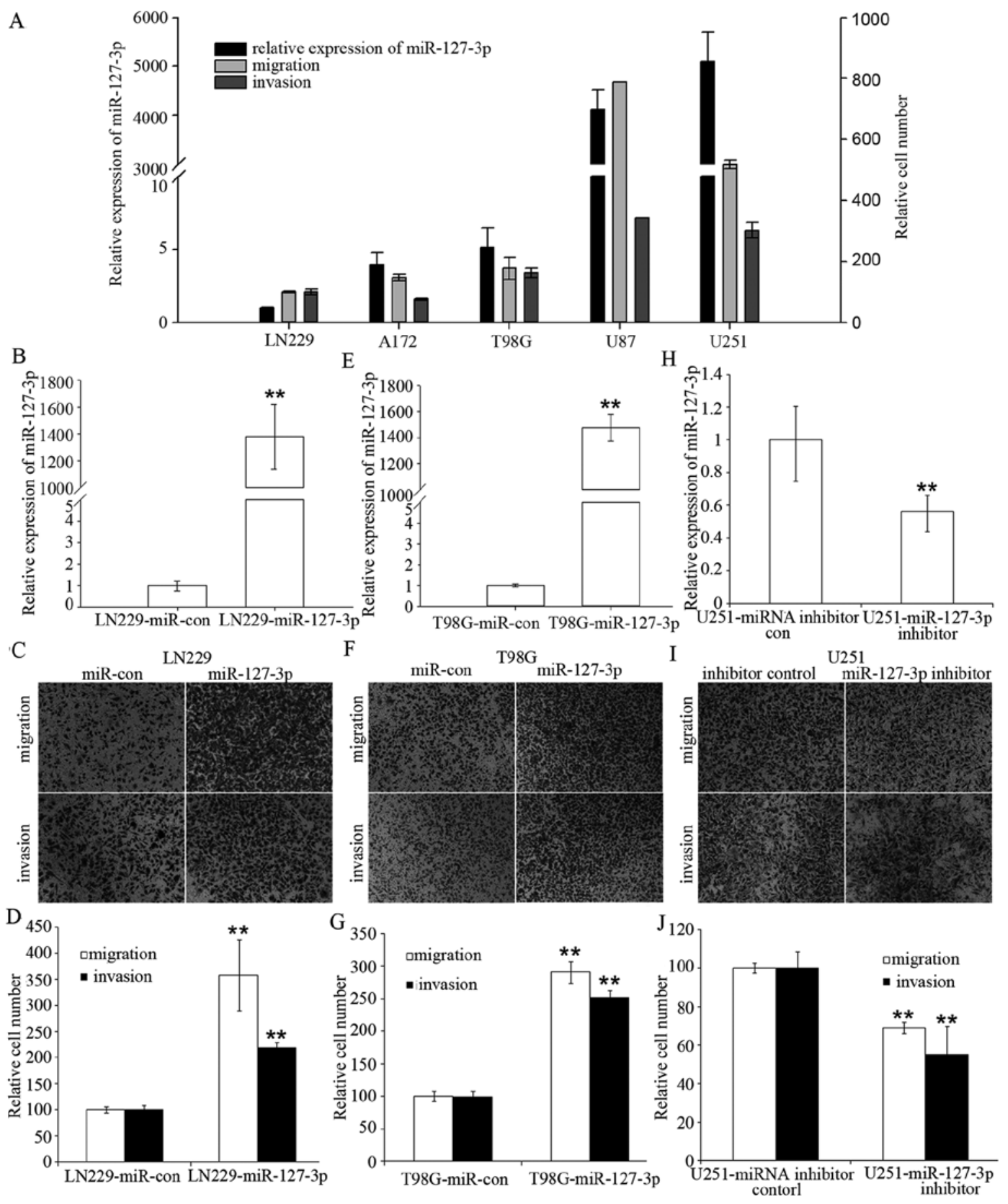

Figure 1. miR-127-3p promotes migration and invasion of human GBM cell lines. (A) miR-127-3p expression (left y-axis) in five GBM cell lines was measured by stem-loop RT-PCR. Migration and invasion potential (right y-axis) was assessed by Transwell assays and quantified by relative cell numbers. Pearson's correlation analysis was used to examine the correlation between miR-127-3p and the migration and invasion potential of human GBM cell lines ( $\mathrm{r}=0.89$ and 0.93 ; $\mathrm{P}<0.05$ ). (B and E) miR-127-3p expression in LN229 and T98G cells stably overexpressing miR-127-3p was detected using stem-loop RT-PCR and the U6 small nuclear RNA was used as an internal control. (C, D, F and G) Migration and invasion abilities of LN229 and T98G cells with stable expression of miR-127-3p or transfected with the control vector were (C and F) assessed by Transwell assays and (D and G) were expressed as relative cell numbers. (H) miR-127-3p expression in U251 cells transfected with miR-127-3p inhibitors or the inhibitor control was assessed by stem-loop RT-PCR. (I and J) Migration and invasion abilities of U251 cells transfected with miR-127-3p inhibitors or inhibitor control were (I) assessed by Transwell assays and (J) were expressed as relative cell numbers. The results are the means of three independent experiments $\pm \mathrm{SD} .{ }^{* *} \mathrm{P}<0.01$.

the pmirGLO-SEPT7-3'UTR but not that of the pmirGLOSEPT7-3'UTR-mut-transfected cells (Fig. 3C).

To test whether miR-127-3p expression affected endogenous SEPT7 expression, we transfected pSUPER.neomiR-127-3p and the control plasmid into LN229 and T98G cells; a decrease in SEPT7 protein level in LN229 and T98G cells was observed (Fig. 4A, the left and the middle panel). Consistent with these results, silencing of miR-127-3p in U251 cells showed an increase in the SEPT7 protein level (Fig. 4A, the right panel). However, the SEPT7 mRNA level was not 

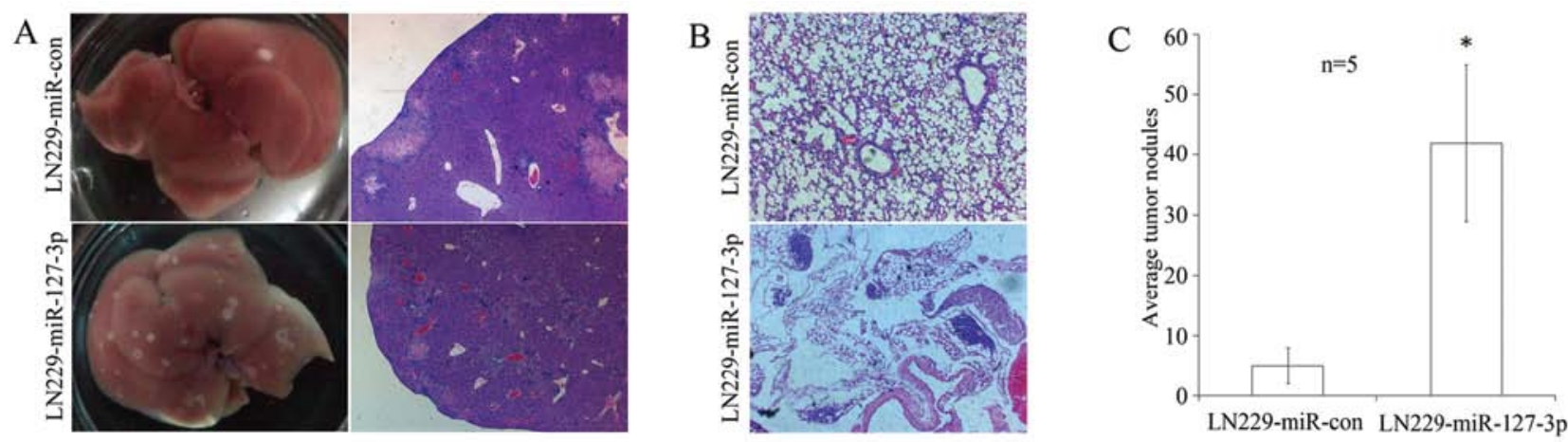

Figure 2. miR-127-3p increases tumor formation in mice. (A) Harvested liver tissues (left) with tumor formation and H\&E staining images (right) of representative sections from mice 8 weeks after injection of LN229 cells stably overexpressing miR-127-3p or transfected with the control vector in nude mice. Original magnification, $x 40$. (B) Representative H\&E staining of lung tumor formation derived from control and miR-127-3p group. Original magnification, $\mathrm{x} 100$. (C) Average numbers of tumor nodules in livers were calculated and graphed. A statistically significant difference in the number of tumor nodules was observed between the control and miR-127-3p group; ${ }^{*} \mathrm{P}<0.05$.

A

\begin{tabular}{|c|c|}
\hline $\begin{array}{l}\text { MiR-127-3p } \\
\text { SEPT7-3'UTR }\end{array}$ & 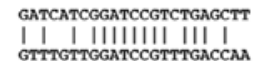 \\
\hline SEPT7-3'UTR-mut & GTTTGTTGGTAGGGTTTGACCAA \\
\hline H.sapiens & GTTTGTTGGATCCGTTTGACCAA \\
\hline P.troglodytes & GITGTIGGATCCGTITGACCAA \\
\hline M.mulatta & GTTTGTIGGATCCGTTTGACCAA \\
\hline R.norvegicus & GTTTGTTGGATCCGTTTGACCAA \\
\hline C.familiaris & GITTGTTGGATCCGITTGACCAA \\
\hline G.gallus & GTTGTIGGATCCGTTGACTAA \\
\hline
\end{tabular}
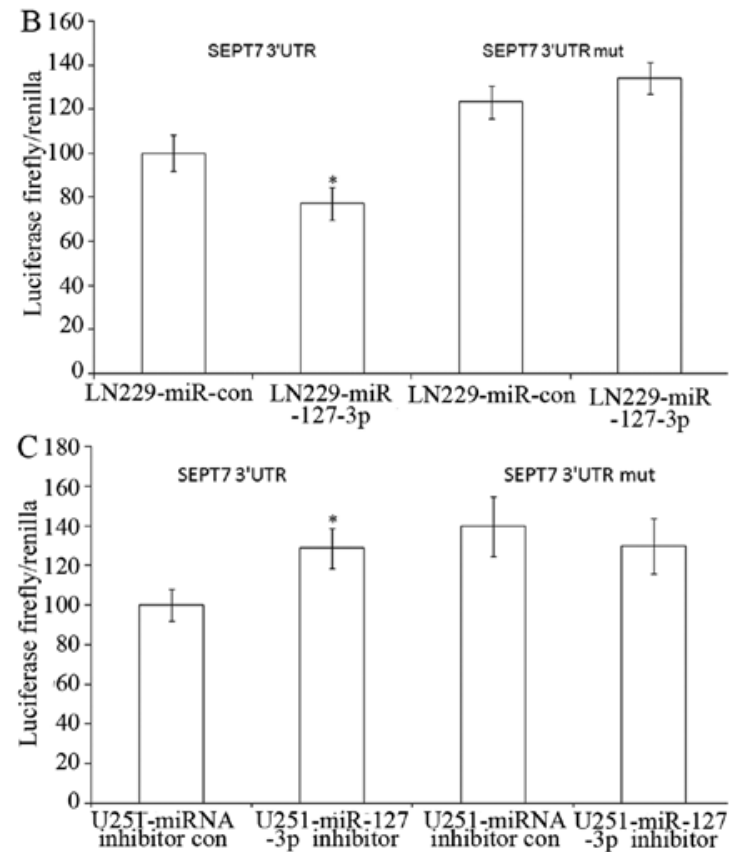

Figure 3. The SEPT7 3'UTR is a target of miR-127-3p. (A) A miR-127-3p target site resides at nucleotides 67-74 of the SEPT7 3'UTR and is highly conserved in different species. Upper panel, sequence alignment of miR-127-3p with binding sites on the SEPT7 3'UTR. pmirGLO-SEPT73'UTR-mut carried a substitution of four nucleotides (bold face) within the miR-127-3p core binding site. Lower panel, sequence of the miR-127-3p core binding site within the 3'UTRs of the SEPT7 genes from six species. (B) Luciferase activity assay demonstrates a direct targeting of the $3^{\prime}$ UTR of SEPT7 by miR-127-3p. LN229 cells were transfected with pSUPER.neomiR-127-3p and pmirGLO-SEPT7-3'UTR/pmirGLO-SEPT7-3'UTR-mut. After 48 h, firefly luciferase activity was measured and normalized to Renilla luciferase activity. (C) U251 cells expressing high levels of endogenous miR-127-3p were transfected with miR-127-3p inhibitor and pmirGLOSEPT7-3'UTR/pmirGLO-SEPT7-3'UTR-mut. After $48 \mathrm{~h}$, firefly luciferase activity was measured and normalized to Renilla luciferase activity. Data are presented as means $\pm \mathrm{SD}, \mathrm{n}=3 ;{ }^{*} \mathrm{P}<0.05$. significantly influenced by overexpression or inhibition of miR-127-3p (Fig. 4B).

We then examined the SEPT7 protein levels in five GBM cell lines (Fig. 4C) and compared them with the miR-127-3p levels. In cell lines with high endogenous miR-127-3p (for example, U87 and U251; Fig. 1A, lanes 4 and 5), a low amount of SEPT7 protein at $49 \mathrm{kDa}$ was observed (Fig. 4C, lanes 4 and 5), whereas cell lines with low miR-127-3p (for example, LN229, A172 and T98G; Fig. 1A, lanes 1-3) showed high amounts of SEPT7 protein (Fig. 4C, lanes 1-3). Across all five cell lines tested, we found a significant inverse correlation $(\mathrm{P}<0.05)$ between miR-127-3p and SEPT7 protein levels (Fig. 4D). We compared the quantified SEPT7 protein expression between the miR-127-3p high expression cell lines (U251 and U87) and low expression cell lines (LN229, A172 and T98 cells) and found that there existed a significant difference between the two groups ( $\mathrm{P}=0.008$; Student's t-test).

We investigated whether EPT7 suppressed cell migration and invasion in LN229 and U251 GBM cells. As shown in Fig. 4E, overexpression of SEPT7 in U251 cells resulted in a decreased cell migration and invasion. To further establish a functional connection between miR-127-3p and SEPT7, we tested whether SEPT7 deregulation was required for miR-127-3p regulation of the migration and invasion of GBM cells. pcDNA3.1-SEPT7, which carried the whole SEPT7 coding sequence without 3'UTR and would thus not be suppressed by the miR-127-3p, and pSUPER.neo-miR-127-3p, were cotransfected into LN229 cells. We found that expression of SEPT7 partly abrogated the migration and invasion initiated by miR-127-3p in the LN229 cells (Fig. 4F).

miR-127-3p regulates genes related to migration and invasion. To identify the global effect of miR-127-3p on GBM cells, we performed microarray analysis to compare the gene expression profile of the miR-127-3p stably overexpressing LN229 cells with that of the LN229 control cells. Among 54,614 probe sets, we identified 1320 (791 downregulated and 529 upregulated) genes that were differentially expressed.

As the U251 GBM cells naturally present higher expression of miR-127-3p (Fig. 1), we compared its expression with LN229, and identified 5280 probe sets with 2-fold changes 

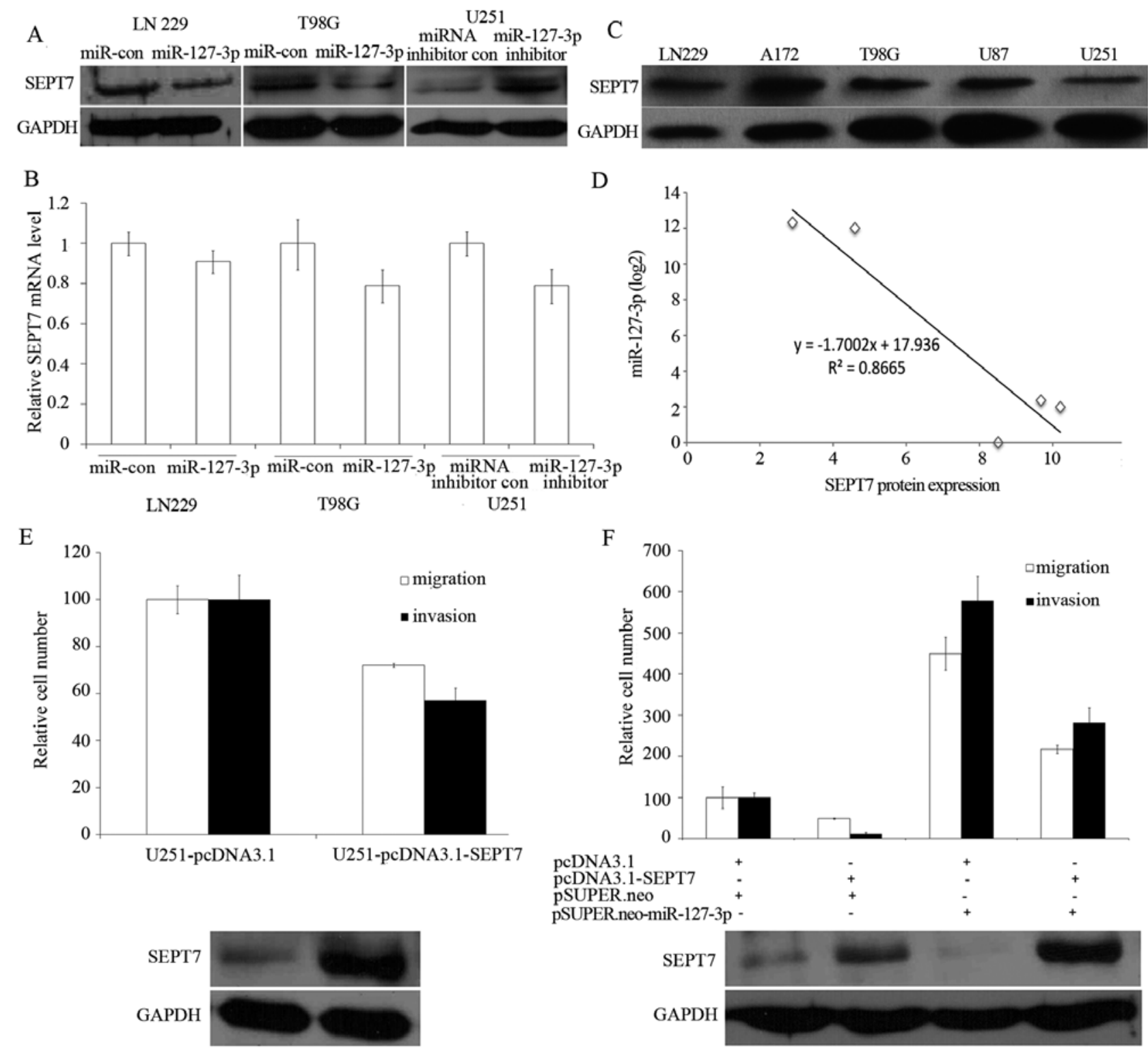

Figure 4. SEPT7 is regulated by miR-127-3p and partly reverses the promotive effects on migration and invasion initiated by miR-127-3p. (A) Western blot analysis of SEPT7 protein expression in LN229 and T98G cells that were transfected with pSUPER.neo-miR-127-3p and control vector, and in U251 cells that were transfected with miR-127-3p inhibitors and inhibitor control. Cell lysates were harvested after $48 \mathrm{~h}$ for the analysis. (B) In parallel, SEPT7 mRNA in the three cell lines treated in A was measured by real-time RT-PCR. GAPDH was used as the internal control. (C) SEPT7 protein level of five GBM cells was assessed by western blot analysis. (D) Inverse correlation between SEPT7 protein expression and miR-127-3p. x-axis, relative SEPT7 protein expression from western blot quantification; $y$-axis, relative miR-127-3p expression ( $\log 2$ transformed from the stem-loop RT-PCR. (E) The migration and invasion potentials of U251 cells that were transfected with pcDNA3.1 and pcDNA3.1-SEPT7. y-axis, relative cell numbers from the Transwell assays after $48 \mathrm{~h}$. (F) The migration and invasion potentials of LN229 cells co-transfected with pcDNA3.1-SEPT7 and pSUPER.neo-miR-127-3p, or their respective control vectors. $y$-axis, relative cell numbers from the Transwell assays after $48 \mathrm{~h}$.

(data not shown). We then intersected the differentially expressed gene list for the comparison between LN229-miR127-3p and LN229 mock control with that for the comparison between U251 and LN229 cells. We identified 855 probe sets that showed differential expression in both comparisons.

Using GO analysis we determined biological process gene categories that were significantly altered by miR-127-3p overexpression (FDR $<0.01$ ). Biological processes including cell proliferation, cell migration, cell motility, regulation of locomotion, which are fundamental biological processes required to achieve cell migration and invasion were found to be enriched (Table I).
miR-127-3p affects remodeling of the actin cytoskeleton by regulating SEPT7 in GBM cells. Dynamic regulation of the filamentous actin (F-actin) cytoskeleton is critical to cell adhesion and migration. We, therefore, examined changes in F-actin remodeling after miR-127-3p expression with a fluorescence microscope. In the control cells, the actin cytoskeleton formed a thick peripheral cytoskeleton near the cell membranes and the cells showed more rounded morphology (Fig. 5A, upper panel), while in the miR-127-3p-overexpressing LN229 GBM cells, the actin cytoskeleton formed thin stress fibers across the cytoplasm and the cells showed spindleshaped morphology (Fig. 5A, lower panel). We then examined 
Table I. miR-127-3p overexpression results in enriched GO categories related to migration and invasion.

\begin{tabular}{|c|c|c|c|c|c|}
\hline GO category & $\begin{array}{c}\text { Total } \\
\text { genes }\end{array}$ & $\begin{array}{l}\text { Changed } \\
\text { genes }\end{array}$ & Enrichment & $\log 10(p)$ & $\begin{array}{c}\text { False } \\
\text { discovery } \\
\text { rate }\end{array}$ \\
\hline GO:0032386_regulation_of_intracellular_transport & 60 & 13 & 5.11 & -6.02 & 0.0000 \\
\hline GO:0033157_regulation_of_intracellular_protein_transport & 53 & 12 & 5.34 & -5.81 & 0.0000 \\
\hline GO:0046822_regulation_of_nucleocytoplasmic_transport & 53 & 12 & 5.34 & -5.81 & 0.0000 \\
\hline GO:0008283_cell_proliferation & 680 & 54 & 1.87 & -5.43 & 0.0000 \\
\hline GO:0016477_cell_migration & 292 & 29 & 2.34 & -4.81 & 0.0000 \\
\hline GO:0046824_positive_regulation_of_nucleocytoplasmic_transport & 29 & 8 & 6.51 & -4.72 & 0.0000 \\
\hline GO:0090316_positive_regulation_of_intracellular_protein_transport & 29 & 8 & 6.51 & -4.72 & 0.0000 \\
\hline GO:0040012_regulation_of_locomotion & 140 & 18 & 3.03 & -4.63 & 0.0000 \\
\hline GO:0032388_positive_regulation_of_intracellular_transport & 30 & 8 & 6.29 & -4.61 & 0.0000 \\
\hline GO:0048870_cell_motility & 301 & 29 & 2.27 & -4.56 & 0.0000 \\
\hline GO:0051674_localization_of_cell & 301 & 29 & 2.27 & -4.56 & 0.0000 \\
\hline GO:0006928_cellular_component_movement & 387 & 34 & 2.07 & -4.42 & 0.0025 \\
\hline GO:0032879_regulation_of_localization & 473 & 39 & 1.94 & -4.37 & 0.0031 \\
\hline GO:0042127_regulation_of_cell_proliferation & 476 & 39 & 1.93 & -4.31 & 0.0029 \\
\hline GO:0002376_immune_system_process & 729 & 53 & 1.71 & -4.25 & 0.0053 \\
\hline GO:0042307_positive_regulation_of_protein_import_into_nucleus & 25 & 7 & 6.60 & -4.24 & 0.0050 \\
\hline GO:0034764_positive_regulation_of_transmembrane_transport & 26 & 7 & 6.35 & -4.12 & 0.0071 \\
\hline GO:0034762_regulation_of_transmembrane_transport & 65 & 11 & 3.99 & -4.11 & 0.0067 \\
\hline GO:0042306_regulation_of_protein_import_into_nucleus & 45 & 9 & 4.72 & -4.04 & 0.0074 \\
\hline GO:0048856_anatomical_structure_development & 1367 & 84 & 1.45 & -3.81 & 0.0085 \\
\hline GO:0008284_positive_regulation_of_cell_proliferation & 253 & 24 & 2.24 & -3.76 & 0.0100 \\
\hline
\end{tabular}

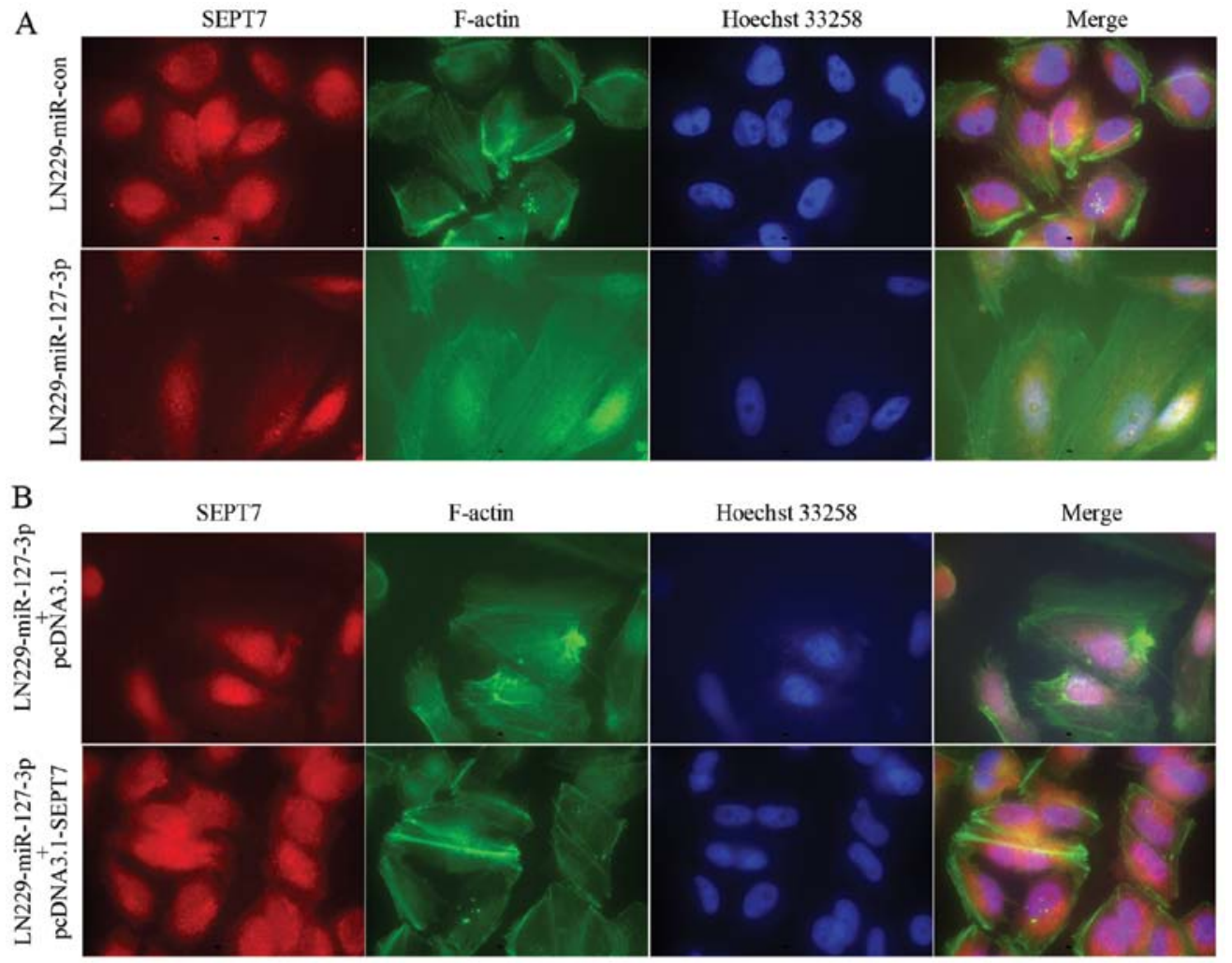

Figure 5. miR-127-3p regulates reorganization of the actin cytoskeleton by SEPT7. (A) Immunofluorescence images of LN229 cells stably overexpressing miR-127-3p or transfected with the control vector following staining with an antibody against SEPT7 and a Cy3-conjugated secondary antibody. Cells were also stained with phalloidin to visualize the actin cytoskeleton. Cell nuclei were stained with Hoechst 33258. (B) Immunofluorescence images of LN229 cells stably overexpressing miR-127-3p transfected with pcDNA3.1 and pcDNA3.1-SEPT7 and stained as A. Original magnification, $\mathrm{x} 1,000$. 
the F-actin remodeling of LN229 cells stably overexpressing miR-127-3p transfected with pcDNA3.1 or pcDNA3.1-SEPT7. The observed changes in cell morphology and the actin cytoskeleton structure in cells overexpressing miR-127-3p were rescued by SEPT7 overexpression (Fig. 5B).

\section{Discussion}

We investigated for the first time the role of miR-127-3p in GBM. Previous studies suggested that miR-127-3p might have tumor suppressing or promotive roles (3-5,7-10). Our inadvertent observation that there existed a statistically significant positive correlation between miR-127-3p expression and the migration and invasion potential of GBM cell lines prompted us to analyze the roles of miR-127-3p in GBM. We showed that miR-127-3p promoted migration and invasion of human GBM cells by in vitro cell analysis, and that miR-127-3p promoted metastasis in in vivo mouse models. This result is consistent with a demonstrated association of miR-127-3p with increases in lymph node metastasis in cervical carcinoma (11). This suggests that miR-127-3p may function as an oncomiR in GBM.

Accumulating evidence suggests that miRNAs play important roles in gliomas including regulation of their migration and invasion (12-14). As initial evidence for a role of miRNA in promoting glioma invasion, Gabriely et al (12) found that miR-21 promotes glioma invasion by targeting MMP inhibitors. Quintavalle et al (15) showed that miR-221/222 overexpession in human GBM increased its invasiveness by targeting the protein phosphate $\mathrm{PTP} \mu$. The inhibitory roles of miRNAs in glioma migration and invasion have also been reported. For example, miR-146b inhibits glioma cell migration and invasion by targeting MMPs (16). Ectopic expression of miR-26b, miR-124a and miR-34a in GBM cell lines resulted in significant inhibition of migration and invasion $(13,17,18)$. miR-7 was downregulated in GBM and it was found to target insulin receptor substrate 2 (IRS2), epidermal growth factor receptor (EGFR) (19) and focal adhesion kinase (FAK). In addition, overexpression of miR-7 resulted in decreased migration and invasion, possibly mediated in part by decreased levels of MMP-2 and MMP-9 (14).

We found that SEPT7 was targeted by miR-127-3p. SEPT7 is a member of the septin family genes. Septins are a group of highly-conserved GTP binding proteins located at the plasma membranes in eukaryote cells (20), and 13 members of septins have been identified in the human (21). Septin members can act as either tumor suppressors or promoters. For example, the expression of ARTS (apoptosis-related protein in the TGF- $\beta$ signaling pathway, the short isoform splice variant SEPT4_v2) is often lost in human leukemia (22). The loss of Sept4 function in mice was found to promote spontaneous leukemia or lymphoma (23). However, SEPT2 was found to be upregulated in human hepatoma carcinoma cells (HCC) and the phosphorylation of Ser218 of SEPT2 is crucial to the proliferation of human hepatoma carcinoma cells (24). SEPT1 plays an important role in spreading in squamous cell carcinoma DJM-1 cells (25). SEPT9 is overexpressed in diverse human tumors including breast, cenral nervous system (CNS), endometrial, kidney, liver, lung, lymphoid, oesophageal, ovarian and pancreatic cancer (26).
The SEPT7 gene is located on human chromosome $7 \mathrm{p} 14.3$, and alternative splicing of the gene results in the transcription of three isoforms. Although SEPT7 is expressed in most tissues, it is especially abundantly expressed in brain tissues (26). SEPT7 is involved in several human diseases including neoplasia $(27,28)$ and neurodegenerative disorders (29). Previous data suggest that SEPT7 is a tumor suppressor. SEPT7 was reported to be downregulated in lowgrade diffuse astrocytomas (30) and in other CNS tumor tissues compared with normal tissues $(26,27)$. In addition, the high level of SEPT7 expression in neuroblastoma and glioma may be associated with favorable clinical and biological characteristics $(27,28)$. SEPT7 has been shown to inhibit the migration and invasion of GBM cell lines (31). This observation indicates that SEPT7 might have a negative role in migration and invasion of GBM cells. Our observation in GBM U251 cells confirmed the suppressive role of SEPT7 on cell migration and invasion (Fig. 4E).

To establish the functional connection between miR-127-3p and SEPT7, we overexpressed SEPT7 and miR-127-3p in LN229 cells and found that SEPT7 protein could partly abrogate the function of miR-127-3p (Fig. 4F). Therefore, SEPT7 protein might be a mediator of the regulation of cell migration and invasion by miR-127-3p. Nonetheless, overexpression of SEPT7 did not completely abolish the effect of miR-127-3p, suggesting that additional mediators might exist. Additional experimentation is necessary to identify these mediators.

Our expression profile analysis of miR-127-3p-regulated genes provided a mechanistic link with its role in promoting migration and invasion as we found that biological processes such as cell migration, cell motility, regulation of locomotion are enriched in miR-127-3p-regulated genes (Table I).

Our data demonstrated that cells with high levels of miR-127-3p exhibited a spindle-shaped morphology and the actin cytoskeleton formed thin stress fibers across the cytoplasm, in contrast with more rounded morphology and a thick peripheral actin cytoskeleton near cell membranes shown in the control cells (Fig. 5). Our data is consistent with reported roles of SEPT7 in cytoskeleton remodeling, cell shape and cell movement (32-35). Recently, SEPT7 was reported to affect the actin cytoskeleton and cellular morphology via the SOCS7/ NCK signaling pathway (29). There is also evidence showing that upregulation of SEPT7 inhibits invasion of GBM cells by distribution of $\alpha$-tubulin (31). Collectively, these data suggest that miR-127-3p regulates cell morphology and the structure of the actin cytoskeleton by targeting SEPT7. Our observation that reintroduction of SEPT7 into LN229 cells overexpressing miR-127-3p rescues the phenotype seems to confirm this hypothesis.

In conclusion, our findings revealed that miR-127-3p promoted the migration and invasion of GBM cells partly by targeting SEPT7. miR-127-3p may be explored as a novel biomarker for predicting the migratory and invasive potential of glioma cells or may be exploited as a therapeutic target for GBM.

\section{Acknowledgements}

This study was funded by grant 81072060 from the National Natural Science Foundation of China; grants 2008DFA11320 and 
2012AA022705 from the Ministry of Science and Technology, China; grant 20110101120153 from the Ministry of Education, China; grant 2012R10021 from the Zhejiang Provincial Government; grant 2011ZX09307-001-05 from National Science and Technology Major Project, China.

\section{References}

1. Huse JT, Holland E and Deangelis LM: Glioblastoma: molecular analysis and clinical implications. Annu Rev Med 64: 59-70, 2013.

2. Filipowicz W, Bhattacharyya SN and Sonenberg N: Mechanisms of post-transcriptional regulation by microRNAs: are the answers in sight? Nat Rev Genet 9: 102-114, 2008.

3. Zhang C, Wang C, Chen X, et al: Expression profile of microRNAs in serum: a fingerprint for esophageal squamous cell carcinoma. Clin Chem 56: 1871-1879, 2010.

4. Mian C, Pennelli G, Fassan M, et al: MicroRNA profiles in familial and sporadic medullary thyroid carcinoma: preliminary relationships with RET status and outcome. Thyroid 22: 890-896, 2012.

5. Saito Y,Liang G, Egger G, et al: Specific activation of microRNA127 with downregulation of the proto-oncogene BCL6 by chromatin-modifying drugs in human cancer cells. Cancer Cell 9: 435-443, 2006.

6. Chen C, Ridzon DA, Broomer AJ, et al: Real-time quantification of microRNAs by stem-loop RT-PCR. Nucleic Acids Res 33: e179, 2005.

7. Yan LX, Huang XF, Shao Q, et al: MicroRNA miR-21 overexpression in human breast cancer is associated with advanced clinical stage, lymph node metastasis and patient poor prognosis. RNA 14: 2348-2360, 2008.

8. Tryndyak VP, Ross SA, Beland FA and Pogribny IP: Downregulation of the microRNAs miR-34a, miR-127, and miR-200b in rat liver during hepatocarcinogenesis induced by a methyldeficient diet. Mol Carcinog 48: 479-487, 2009.

9. Pan C, Chen H, Wang L, et al: Down-regulation of miR-127 facilitates hepatocyte proliferation during rat liver regeneration. PLoS One 7: e39151, 2012.

10. Dixon-McIver A, East P, Mein CA, et al: Distinctive patterns of microRNA expression associated with karyotype in acute myeloid leukaemia. PLoS One 3: e2141, 2008.

11. Lee JW, Choi CH, Choi JJ, et al: Altered microRNA expression in cervical carcinomas. Clin Cancer Res 14: 2535-2542, 2008.

12. Gabriely G, Wurdinger T, Kesari S, et al: MicroRNA 21 promotes glioma invasion by targeting matrix metalloproteinase regulators. Mol Cell Biol 28: 5369-5380, 2008.

13. Guessous F, Zhang Y, Kofman A, et al: microRNA-34a is tumor suppressive in brain tumors and glioma stem cells. Cell Cycle 9: 1031-1036, 2010.

14. Wu DG, Wang YY, Fan LG, et al: MicroRNA-7 regulates glioblastoma cell invasion via targeting focal adhesion kinase expression. Chin Med J (Engl) 124: 2616-2621, 2011.

15. Quintavalle C, Garofalo M, Zanca C, et al: miR-221/222 overexpession in human glioblastoma increases invasiveness by targeting the protein phosphate PTPmu. Oncogene 31: 858-868, 2012 .
16. Xia H, Qi Y, Ng SS, et al: microRNA-146b inhibits glioma cell migration and invasion by targeting MMPs. Brain Res 1269: 158-165, 2009.

17. Wu N, Zhao X, Liu M, et al: Role of microRNA-26b in glioma development and its mediated regulation on EphA2. PLoS One 6: e16264, 2011.

18. Fowler A, Thomson D, Giles K, et al: miR-124a is frequently down-regulated in glioblastoma and is involved in migration and invasion. Eur J Cancer 47: 953-963, 2011.

19. Kefas B, Godlewski J, Comeau L, et al: microRNA-7 inhibits the epidermal growth factor receptor and the Akt pathway and is down-regulated in glioblastoma. Cancer Res 68: 3566-3572, 2008.

20. Mostowy S and Cossart P: Septins: the fourth component of the cytoskeleton. Nat Rev Mol Cell Biol 13: 183-194, 2012.

21. Hall PA and Russell SE: The pathobiology of the septin gene family. J Pathol 204: 489-505, 2004.

22. Elhasid R, Sahar D, Merling A, et al: Mitochondrial pro-apoptotic ARTS protein is lost in the majority of acute lymphoblastic leukemia patients. Oncogene 23: 5468-5475, 2004.

23. Garcia-Fernandez M, Kissel H, Brown S, et al: Sept4/ARTS is required for stem cell apoptosis and tumor suppression. Genes Dev 24: 2282-2293, 2010.

24. Yu W, Ding X, Chen F, et al: The phosphorylation of SEPT2 on Ser218 by casein kinase 2 is important to hepatoma carcinoma cell proliferation. Mol Cell Biochem 325: 61-67, 2009.

25. Mizutani Y, Ito H, Iwamoto I, et al: Possible role of a septin, SEPT1, in spreading in squamous cell carcinoma DJM-1 cells. Biol Chem 394: 281-290, 2013.

26. Scott M, Hyland PL, McGregor G, Hillan KJ, Russell SE and Hall PA: Multimodality expression profiling shows SEPT9 to be overexpressed in a wide range of human tumours. Oncogene 24: 4688-4700, 2005.

27. Jia ZF, Huang Q, Kang CS, et al: Overexpression of septin 7 suppresses glioma cell growth. J Neurooncol 98: 329-340, 2010.

28. Nagata T, Takahashi Y, Asai S, et al: The high level of hCDC10 gene expression in neuroblastoma may be associated with favorable characteristics of the tumor. J Surg Res 92: 267-275, 2000.

29. Kremer BE, Adang LA and Macara IG: Septins regulate actin organization and cell-cycle arrest through nuclear accumulation of NCK mediated by SOCS7. Cell 130: 837-850, 2007.

30. Huang H, Colella S, Kurrer M, Yonekawa Y, Kleihues P and Ohgaki H: Gene expression profiling of low-grade diffuse astrocytomas by cDNA arrays. Cancer Res 60: 6868-6874, 2000.

31. Xu S, Jia ZF, Kang C, et al: Upregulation of SEPT7 gene inhibits invasion of human glioma cells. Cancer Invest 28: 248-258, 2010.

32. Nagata K, Asano T, Nozawa Y and Inagaki M: Biochemical and cell biological analyses of a mammalian septin complex, Sept7/9b/11. J Biol Chem 279: 55895-55904, 2004

33. Bowen JR, Hwang D, Bai X, Roy D and Spiliotis ET: Septin GTPases spatially guide microtubule organization and plus end dynamics in polarizing epithelia. J Cell Biol 194: 187-197, 2011.

34. Kim SK, Shindo A, Park TJ, et al: Planar cell polarity acts through septins to control collective cell movement and ciliogenesis. Science 329: 1337-1340, 2010.

35. Tada T, Simonetta A, Batterton M, Kinoshita M, Edbauer D and Sheng M: Role of Septin cytoskeleton in spine morphogenesis and dendrite development in neurons. Curr Biol 17: 1752-1758, 2007. 